\title{
Association of COVID-19-Related Hospital Use and Overall COVID-19 Mortality in the USA
}

$\mathrm{J}$ Gen Intern Med

DOI: $10.1007 / \mathrm{s} 11606-020-06084-7$

(C) Society of General Internal Medicine 2020

\section{BACKGROUND}

Most policy interventions to address the COVID-19 outbreak in the USA have focused on "flattening the curve"-an approach to spread out hospitalizations over a longer duration to avoid overwhelming the healthcare system. ${ }^{1}$ The forecasting models informing these policy decisions make assumptions about the relation between the number of COVID-19 cases, hospitalizations, intensive care unit (ICU) demand, and subsequent impact on mortality. ${ }^{2}$

\section{OBJECTIVE}

To quantify the association of daily ICU and non-ICU hospital use by COVID-19 patients on overall COVID-19 mortality.

\section{METHODS}

We used data from the University of Minnesota COVID-19 Hospitalization Tracking Project ${ }^{3}$ from the 23 states who reported daily data on current COVID-19 hospitalized patients in ICU and non-ICU beds between March 20 and June 4. The unit of observation was a state-day. The outcome variable was defined as the number of COVID-19 deaths over the next 7 days, including the current day. The exposure variables were current ICU use and non-ICU use. The ICU use was calculated as the number of current COVID-19 ICU patients expressed as a percentage of the total ICU beds. ${ }^{4}$ The non-ICU use was calculated similarly. We used generalized estimating equations models with a negative binomial distribution, log link, and an autoregressive one-error structure. The model adjusted for the 3-day lags of ICU use and non-ICU use, as well as the cumulative number of cases, ${ }^{5}$ incident cases in the last 7 days, indicators for the state, week of the year, and day of the week. We reported adjusted changes in COVID-19 deaths over the next 7 days when either ICU or non-ICU use exposure variable increased by $1 \%$ point. Robust standard errors and $95 \%$

Received June 20, 2020

Accepted July 27, 2020 confidence intervals were reported. All analyses were conducted using STATA v16.0.

\section{FINDINGS}

There were a total of 1056 observations across the 23 states during the study period. The average COVID-19-related ICU use was $19.6 \%$ (SD 19.2); non-ICU use was 5.5\% (SD 6.3). In adjusted analyses, our base model indicated that a $1 \%$ point increase in ICU bed use (about 17 ICU beds on average) is associated with a $2.84(p=0.01,95 \%$ CI $[.6,5])$ increase in COVID-19 deaths over the next 7 days (Table 1, model 1). The corresponding association for a $1 \%$ point increase in nonICU bed use (about 130 non-ICU beds on average) was 17.84 more COVID-19 deaths ( $p<0.001,95 \%$ CI $[11.1,24.6])$. The results remain robust across a variety of alternate specifications (Table 1, models 2-7).

Figure 1 plots the association at different levels of ICU and non-ICU use. For example, when COVID-19 patients occupy $25 \%$ of all ICU beds in a state, a $1 \%$ point increase in ICU bed use would lead to 2.3 ( $p=0.002 ; 95 \%$ CI $0.8,3.8$ ) additional COVID-19 deaths over the next 7 days. Similarly, when COVID-19 patients occupy $9 \%$ of all non-ICU beds in a state, a $1 \%$ point increase in ICU bed use would lead to $15.3(p<$ $0.001 ; 95 \%$ CI 9.6, 21.0) additional COVID-19 deaths over the next 7 days.

\section{DISCUSSION}

ICU and non-ICU bed use as a percentage of the bed capacity of a state were significantly associated with the increase of overall COVID-19 deaths over the next 7 days. The intensity of association for ICU bed use was higher than for non-ICUbed use. One limitation of our study is that during the COVID19 era, some states may have been able to expand their hospital beds. However, doing so for ICU beds was likely difficult due to infrastructure constraints. Our study quantifies the relationship between COVID-19 deaths and COVID-19 hospitalizations using actual data. These estimates provide a better understanding of the projections of the COVID-19 pandemic in the USA especially when states are gearing up to restart economic activities and provide important practice insights for hospitals in terms of assessment of hospital bed and ICU bed capacity and preparedness. 
Table 1 Adjusted Change [95\% CI] in COVID-19 Deaths over the Next 7 Days, Including Present Day (Days 0 to 6)

\begin{tabular}{|c|c|c|c|c|c|c|c|}
\hline & Model 1 (base) & Model 2 & Model 3 & Model 4 & Model 5 & Model 6 & Model 7 \\
\hline COVID-19 ICU use* & 2.84 & 2.71 & 1.79 & 2.48 & 2.46 & 2.99 & $\begin{array}{ll}3.31 & \\
& \end{array}$ \\
\hline COVID-19 non-ICU use* & $\begin{array}{l}{[0.6,5]} \\
17.84 \\
{[11.1,24.6]}\end{array}$ & $\begin{array}{l}{[0.4,5]} \\
17.23 \\
{[10.1,24.4]}\end{array}$ & $\begin{array}{l}{[0.7,2.9]} \\
18.89 \\
{[12.3,25.5]}\end{array}$ & $\begin{array}{l}{[1.3,3.7]} \\
6.15 \\
{[2.2,10.1]}\end{array}$ & $\begin{array}{l}{[1.3,3.6]} \\
6.41 \\
{[2.7,10.2]}\end{array}$ & $\begin{array}{l}{[0.7,5.3]} \\
17.81 \\
{[11,24.6]}\end{array}$ & $\begin{array}{l}{[0.9,5.7]} \\
17.37 \\
{[10.7,24.1]}\end{array}$ \\
\hline 3-day lags of COVID-19 ICU use & Yes & Yes & No & No & No & Yes & Yes \\
\hline 3-day lags of COVID-19 non-ICU use & Yes & Yes & Yes & No & No & Yes & Yes \\
\hline Cumulative \# of cases & Yes & Yes & Yes & Yes & Yes & Yes & Yes \\
\hline Cases in days 0 to -6 & Yes & No & Yes & Yes & No & Yes & Yes \\
\hline Week of year indicators & Yes & Yes & Yes & Yes & Yes & Yes & Yes \\
\hline Day of week indicators & Yes & Yes & Yes & Yes & Yes & Yes & Yes \\
\hline State indicators & Yes & Yes & Yes & Yes & Yes & Yes & Yes \\
\hline Estimator & GEE & GEE & GEE & GEE & GEE & GEE & GEE \\
\hline \multirow[t]{2}{*}{ Specification } & Log link & Log link & Log link & Log link & Log link & Log link & Log link \\
\hline & Neg Bin & Neg Bin & Neg Bin & Neg Bin & Neg Bin & Neg Bin & Neg Bin \\
\hline$N$ & 1056 & 1062 & 1056 & 1118 & 1131 & 1056 & 1056 \\
\hline \# Of states & 23 & 23 & 23 & 23 & 23 & 23 & 23 \\
\hline Error correlation & AR1 & AR1 & AR1 & AR1 & AR1 & AR2 & AR3 \\
\hline Standard errors & Robust & Robust & Robust & Robust & Robust & Robust & Robust \\
\hline
\end{tabular}

Notes: In our analysis sample, mean (SD, 25th percentile-75th percentile) of key variables were the following: COVID-19 ICU use was 19.6 (19.22, 7.8-22.1); COVID-19 non-ICU use was 5.5 (6.3, 1.5-8.8); total ICU bed capacity, 1678 (1677, 643-1891); total non-ICU bed capacity, 12,978 (12,418, 5581-15,462); COVID-19 deaths over the next 7 days, 234 (358, 40-276)

Minimum number of days of data contributed by a state was 6. Maximum number was 65

GEE, generalized estimating equation; AR1, autoregressive one-error structure (also known as first-order autoregressive process); AR2 and AR3 are similarly defined

*The ICU use was calculated as the number of current ICU patients with COVID-19 and then expressed as a percentage of the total ICU beds of that state. The non-ICU use was calculated similarly
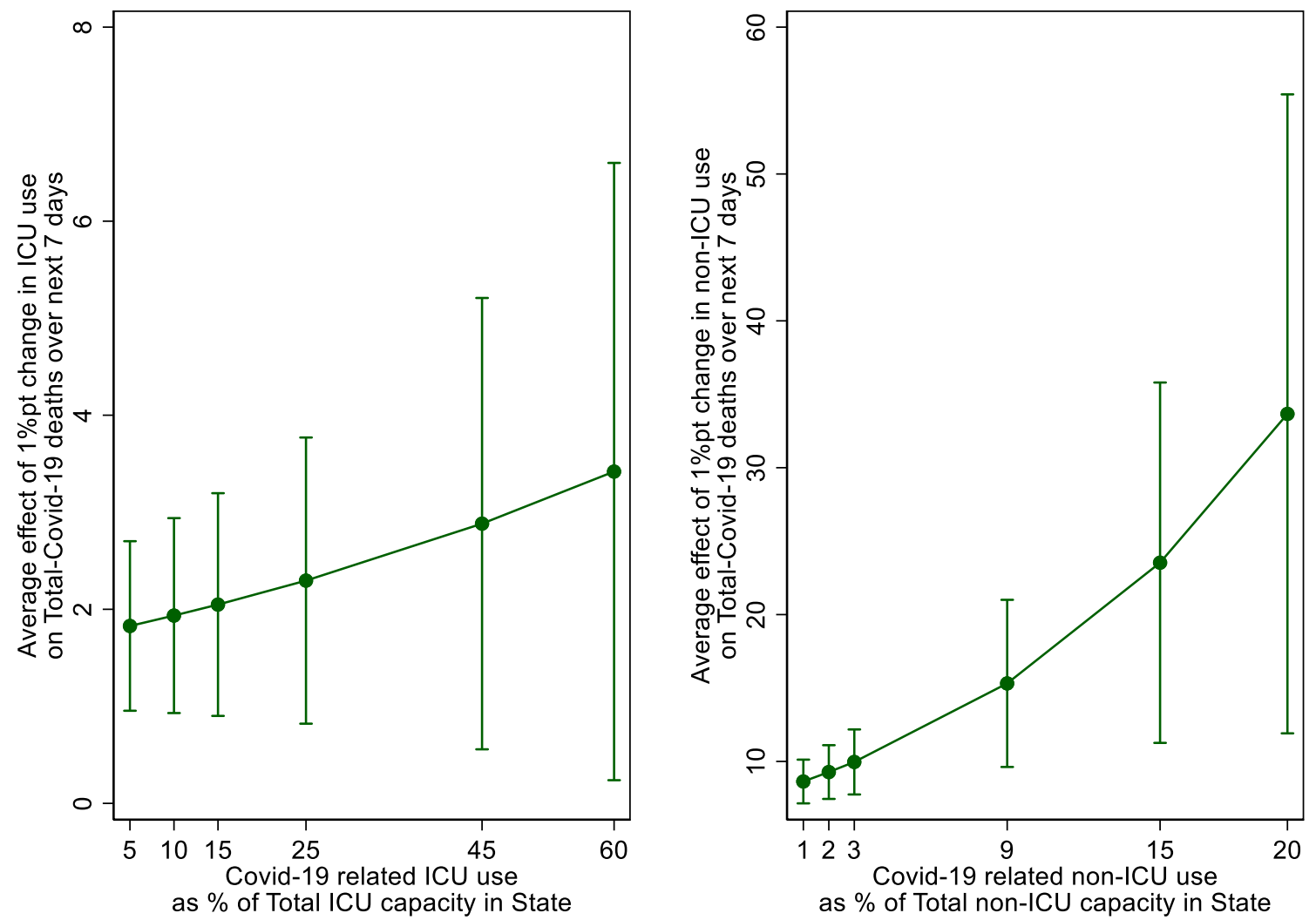

Figure 1 Adjusted average association of $1 \%$ point increase in ICU or non-ICU use by COVID-19 patients on counts of COVID-19 deaths over the next 7 days, by levels of use. Note: Each point corresponds to closely represent the 15th, 25th, 50th, 75th, 90th, and 95th percentile of the ICU use (left) and non-ICU (right) on their corresponding distribution in our analysis sample. 
Acknowledgments: Pinar Karaca-Mandic and Anirban Basu had full access to all the data in the study and take responsibility for the integrity of the data and the accuracy of the data analysis. This research uses publicly available data from the University of Minnesota COVID-19 Hospitalization Project, which is partially funded by the University of Minnesota Office of Academic Clinical Affairs and the United Health Foundation.

Pinar Karaca-Mandic, $P h D^{1}$

Soumya Sen, $\mathrm{Ph} \mathrm{D}^{2}$

Archelle Georgiou, $M D^{3}$

Yi Zhu, $M S^{2}$

Anirban Basu, $\mathrm{PhD}^{4}$

${ }^{1}$ Department of Finance, University of Minnesota Carlson School of Management,

Minneapolis, MN, USA

${ }^{2}$ Department of Information \& Decision Sciences, University of Minnesota Carlson School of Management,

Minneapolis, MN, USA

${ }^{3}$ Starkey Hearing Technologies,

Eden Prairie, MN, USA

${ }^{4}$ Departments of Pharmacy, Health Services, and Economics University of Washington,

Seattle, WA, USA

Corresponding Author: Pinar Karaca-Mandic, $\mathrm{PhD}$; Department of Finance, University of Minnesota Carlson School of Management, Minneapolis, MN, USA (e-mail: pkmandic@umn.edu).

Funding Information This research is partially funded by the University of Minnesota Office of Academic Clinical Affairs and the United Health Foundation.

\section{Compliance with Ethical Standards:}

Conflict of Interest: Dr. Karaca-Mandic reports funding from the University of Minnesota, Office of Academic Clinical Affairs and grants from United Health Foundation during the conduct of the study; personal fees from Tactile Medical, personal fees from Precision Health Economics, personal fees from Sempre Health, grants from Agency for Healthcare Research and Quality, grants from American Cancer Society, grants from National Institute for Health Care Management, grants from National Institute on Drug Abuse, grants from National Institutes of Health outside the submitted work. Dr. Georgiou reports personal fees from HealthGrades outside the submitted work. Dr. Basu reports personal fees from Salutis Consulting LLC outside of submitted work.

Disclaimer: These organizations had no role in the design and conduct of the study; collection, management, analysis, and interpretation of the data; preparation, review, or approval of the manuscript; and decision to submit the manuscript for publication.

\section{REFERENCES}

1. Desai A. Twentieth-Century Lessons for a Modern Coronavirus Pandemic. JAMA. 2020;323(21):2118-2119. https://doi.org/10.1001/jama.2020. 4165

2. Forecasting the impact of the first wave of the COVID-19 pandemic on hospital demand and deaths for the USA and European Economic Area countries, IHME COVID-19 health service utilization forecasting team, Christopher JL Murray medRxiv 2020.04.21.20074732; https://doi.org/ 10.1101/2020.04.21.20074732, [https://www.medrxiv.org/content/10. 1101/2020.04.21.20074732v1, Last Accessed Jun 4, 2020]

3. University of Minnesota COVID-19 Hospitalization Tracking Project website. [https://carlsonschool.umn.edu/mili-misrc-covid 19-trackingproject, Last accessed Jun 4, 2020]

4. Harvard Global Health Institute, U.S. Hospital Capacity. Total hospital beds and ICU beds were retrieved on March 27, 2020 from https:// globalepidemics.org/our-data/hospital-capacity/

5. New York Times Covid-19 Data Repository. Data on confirmed cases were retrieved on June 6, 2020 from https://github.com/nytimes/covid-19data

Publisher's Note: Springer Nature remains neutral with regard to jurisdictional claims in published maps and institutional affiliations. 